\title{
CONGRUENCE INTERSECTION PROPERTIES FOR VARIETIES OF ALGEBRAS
}

\author{
PAOLO AGLIANO and KIRBY A. BAKER
}

(Received 27 October 1997; revised 16 April 1999)

Communicated by B. A. Davey

\begin{abstract}
It is shown that a variety $\mathcal{V}$ has distributive congruence lattices if and only if the intersection of two principal congruence relations is definable by equations involving terms with parameters. The nature of the terms involved then provides a useful classification of congruence distributive varieties. In particular, the classification puts into proper perspective two stronger properties. A variety is said to have the Principal Intersection Property if the intersection of any two principal congruence relations is principal, or the Compact Intersection Property if the intersection of two compact congruence relations is compact. For non-congruence-distributive varieties, it is shown that some useful constructions are nevertheless possible.
\end{abstract}

1991 Mathematics subject classification (Amer. Math. Soc.): primary 08A30, 08B10. Keywords and phrases: principal intersection property, compact intersection property.

\section{Introduction}

For congruence-distributive varieties of algebras, the importance of the structure of the intersection of two principal congruence relations has long been evident $[4,5]$. In particular, many such varieties, especially some familiar from algebraic logic, satisfy one or both of these two strong properties: A variety $\mathcal{V}$ has the principal intersection property (PIP) if in each algebra in $\mathcal{V}$ the intersection of any two principal congruences is principal, or the compact intersection property (CIP) if the intersection of any two compact congruences is compact [4]. Blok and Pigozzi [9] have shown that, for a variety, the CIP and congruence distributivity together are equivalent to the definability of the intersection of two principal congruences by finitely many equations without parameters.

(C) 1999 Australian Mathematical Society $0263-6115 / 99 \$ A 2.00+0.00$ 
In Section 2 it is shown that, for a variety, congruence distributivity itself is equivalent to the definability of the intersection of two principal congruence relations by equations (perhaps infinitely many), possibly with parameters. This perspective yields a classification of congruence-distributive varieties into four categories, one of which consists of varieties with the CIP. Section 3 shows that, nevertheless, the PIP and the CIP have some applicability for non-congruence-distributive varieties; a lifting theorem for reducts provides a useful construction. In Section 4 , this construction is seen to yield more detailed information in the congruence distributive case, with some instructive examples.

General references are $[13,15,18]$. The principal congruence relation generated by identifying $a, b \in A$ will be denoted by $\vartheta_{\mathrm{A}}(a, b)$. Compact congruence relations can be characterized as those that are a join of finitely many principal congruence relations. If a variety has the CIP, then the compact congruences of any member of the variety form a sublattice of the congruence lattice. Note that the CIP and the PIP are not as close in concept as it may seem; the CIP is purely lattice-theoretic and depends only on the congruence lattice up to lattice isomorphism, while the PIP may depend on the specific operations of the variety (Example 4.4 and Example 4.7).

This project originated from a search by the first author for examples of congruence distributive varieties with the CIP but not the PIP. Several such examples are presented in this paper.

\section{Congruence distributivity and intersection properties of congruences}

Let $\mathcal{V}$ be any variety. A system of congruence intersection terms with parameters for $\mathcal{V}$ is a family $\left\{\left(p_{i}, q_{i}\right): i \in I\right\}$ of pairs of terms in variables $x, y, z, w, u_{0}, u_{1}, \ldots$ such that for every $\mathbf{A} \in \mathcal{V}$ and $a, b, c, d \in A$

$$
\vartheta_{\mathbf{A}}(a, b) \cap \vartheta_{\mathbf{A}}(c, d)=\bigvee_{\mathbf{e} \in A^{\omega}} \bigvee_{i \in I} \vartheta_{\mathbf{A}}\left(p_{i}(a, b, c, d, \mathbf{e}), q_{i}(a, b, c, d, \mathbf{e})\right)
$$

where $p_{i}(a, b, c, d, \mathbf{e})$ denotes $p_{i}\left(a, b, c, d, e_{0}, \ldots, e_{n_{i}}\right)$ and similarly for $q_{i}$. Thus in the presence of such a system, the intersection of two principal congruence relations can be described in an explicit uniform way. It will be shown that the existence of such a system is equivalent to congruence distributivity (Theorem 2.4 ).

In a system of congruence intersection terms, it may be possible to choose $I$ finite or not, and the parameters may be needed or not. Thus we obtain a four-way perspective on congruence distributive varieties, depicted schematically in Figure 1, which embodies a number of assertions to be justified below.

In this section, for the free algebra on generators $x, y, z, \ldots$ let us write $\mathbf{F}(x, y$, $z, \ldots)$. For a surjection $\phi: \mathbf{A} \rightarrow \mathbf{B}$, let $\phi^{*}: \operatorname{Con}(\mathbf{A}) \rightarrow \operatorname{Con}(\mathbf{B})$ be the induced 


\begin{tabular}{l|l|l} 
finite & $\begin{array}{l}\text { without parameters } \\
\text { Any congruence distributive } \\
\text { (Corollary } 2.7)\end{array}$ & $\begin{array}{l}\text { Any variety generated by } \\
\text { a finite nondistributive lattice. } \\
\text { (Example 2.9) }\end{array}$ \\
\hline infinite & $\begin{array}{l}\text { The variety of Ockham algebras. } \\
\text { (Example 4.3) }\end{array}$ & $\begin{array}{l}\text { The variety of all lattices. } \\
\text { (Example 2.10) }\end{array}$
\end{tabular}

FIGURE 1. The four-way classification

map on congruence lattices. As observed in [4, Lemma 1.11], $\phi^{*}$ preserves arbitrary joins and principal congruences, and in the presence of congruence distributivity preserves (finite) meets as well. The next lemma characterizes systems of congruence intersection terms with parameters.

LEMMA 2.1. (But see also [4].) Let $\mathcal{V}$ be a congruence distributive variety and let $\left\{\left(p_{i}, q_{i}\right): i \in I\right\}$ be a family of pairs of terms in variables $x, y, z, w, u_{0}, u_{1}, \ldots$ Then the following are equivalent.

(1) The family is a system of congruence intersection terms with parameters for $\mathcal{V}$.

(2) In any subdirectly irreducible algebra $\mathbf{A} \in \mathcal{V}$, for any $a, b, c, d \in A$,

$$
\begin{aligned}
& p_{i}(a, b, c, d, \mathbf{e})=q_{i}(a, b, c, d, \mathbf{e}) \quad \text { for all } i \in I \text { and all } \mathbf{e} \in A^{\omega} \\
& \text { if and only if } a=b \text { or } c=d .
\end{aligned}
$$

PROOF. That (1) implies (2) is evident from the fact that $0_{\mathbf{A}} \in \operatorname{Con}(\mathbf{A})$ is meetirreducible. To verify that (2) implies (1), let $\mathbf{A} \in \mathcal{V}$ and $a, b, c, d, e_{0}, e_{1}, \ldots \in A$. Since $\operatorname{Con}(\mathbf{A})$ is an algebraic lattice, any congruence is the intersection of completely meet irreducible congruences. Thus it is enough to show that, if $\varphi$ is completely meet irreducible in $\operatorname{Con}(\mathbf{A})$,

$$
\begin{aligned}
& \vartheta_{\mathrm{A}}(a, b) \cap \vartheta_{\mathrm{A}}(c, d) \leq \varphi \quad \text { if and only if } \\
& \vartheta_{\mathrm{A}}\left(p_{i}(a, b, c, d, \mathbf{e}), q_{i}(a, b, c, d, \mathrm{e})\right) \leq \varphi, \quad i \in I, \mathbf{e} \in A^{\omega} .
\end{aligned}
$$

Let $\mathbf{B}=\mathbf{A} / \varphi$. Then $\mathbf{B}$ is subdirectly irreducible and, since $\mathcal{V}$ is congruence distributive, the equivalence above becomes

$$
\begin{aligned}
& \vartheta_{\mathbf{B}}(a / \varphi, b / \varphi) \cap \vartheta_{\mathbf{B}}(c / \varphi, d / \varphi)=0_{\mathbf{B}} \quad \text { if and only if } \\
& \vartheta_{\mathbf{B}}\left(p_{i}(a, b, c, d, \mathbf{e}) / \varphi, q_{i}(a, b, c, d, \mathbf{e}) / \varphi\right)=0_{\mathbf{B}}, \quad i \in I, \mathbf{e} \in A^{\omega} .
\end{aligned}
$$

Since $\mathbf{B} \in \mathcal{V}$ this holds by hypothesis. 
COROLLARY 2.2. Under the equivalent conditions of Lemma 2.1, an algebra $\mathbf{A}$ is finitely subdirectly irreducible if and only if condition (2.2) holds.

PROOF. Finite subdirect irreducibility is equivalent to the condition that $0_{\mathbf{A}} \in$ Con(A) be meet-irreducible, which is in turn equivalent to the condition that $\vartheta_{\mathbf{A}}(a, b) \cap$ $\vartheta_{\mathrm{A}}(c, d)=0_{\mathrm{A}}$ if and only if $\vartheta_{\mathrm{A}}(a, b)=0$ or $\vartheta_{\mathrm{A}}(c, d)=0$, an assertion expressed by condition (2.2).

LEMMA 2.3. Let $\mathcal{V}$ be a congruence distributive variety and consider a family of pairs of terms $\left\{\left(p_{i}, q_{i}\right): i \in I\right\}$ in variables $x, y, z, w, u_{0}, u_{1}, \ldots$ If in the free algebra $\mathbf{F}=\mathbf{F}\left(x, y, z, w, u_{0}, u_{1}, \ldots\right)$, the family describes $\vartheta_{\mathbf{F}}(x, y) \cap \vartheta_{\mathbf{F}}(z, w)$, without needing quantification over parameters,

$$
\vartheta_{\mathbf{F}}(x, y) \cap \vartheta_{\mathbf{F}}(z, w)=\bigvee_{i \in I} \vartheta_{\mathbf{F}}\left(p_{i}(x, y, z, w, \mathbf{u}), q_{i}(x, y, z, w, \mathbf{u})\right),
$$

then the family is a system of congruence intersection terms with parameters for $\mathcal{V}$.

PROOF. Regard (2.1) as a pair of inclusions. For the right-to-left inclusion, observe that the following laws hold for each $i \in I$ :

$$
\begin{aligned}
p_{i}\left(x, x, z, w, u_{0}, \ldots, u_{n_{i}}\right) & \approx q_{i}\left(x, x, z, w, u_{0}, \ldots, u_{n_{i}}\right), \\
p_{i}\left(x, y, z, z, u_{0}, \ldots, u_{n_{i}}\right) & \approx q_{i}\left(x, y, z, z, u_{0}, \ldots, u_{n_{i}}\right) .
\end{aligned}
$$

The first of these laws can be verified by mapping $\mathbf{F}$ onto a free subalgebra of $\mathbf{F}$ by sending $y$ to $x$ and other generators to themselves; the second law is similarly derived.

To verify the opposite inclusion in (2.1), first consider the case where $\mathbf{A} \in \mathcal{V}$ is countably generated, say by $e_{0}, e_{1}, \ldots$ Then we can map $x \mapsto a, y \mapsto b, z \mapsto c$, $w \mapsto d$, and $u_{j} \mapsto e_{j}$ to get a surjection $\phi: \mathbf{F} \rightarrow \mathbf{A}$. Since $\phi^{*}$ is a join-complete lattice homomorphism preserving principal congruences, the left-to-right inclusion in $(2,1)$ holds even without quantifying over parameters.

For arbitrary A observe that, by the Mal'tsev construction of principal congruences, any inclusion $\vartheta_{\mathbf{A}}(r, s) \subseteq \vartheta_{\mathbf{A}}(a, b) \cap \vartheta_{\mathbf{A}}(c, d)$ can be verified within a finitely (hence countably) generated subalgebra of $\mathbf{A}$. It follows that

$$
\vartheta_{\mathbf{A}}(r, s) \subseteq \bigvee_{\mathbf{e} \in A^{\omega}} \bigvee_{i \in l} \vartheta_{\mathbf{A}}\left(p_{i}(a, b, c, d, \mathbf{e}), q_{i}(a, b, c, d, \mathbf{e})\right)
$$

THEOREM 2.4. For a variety $\mathcal{V}$ the following are equivalent.

(1) $V$ is congruence distributive.

(2) $\mathcal{V}$ has a system of congruence intersection terms with parameters. 
PROOF. As already remarked, [4, Theorem 3.4] proves the implication from (1) to (2) An alternative proof is immediate from Lemma 2.3: In the presence of congruence distributivity, merely expressing $\vartheta(x, y) \cap \vartheta(z, w)$ as a join of principal congruences in $\mathbf{F}\left(x, y, z, w, u_{0}, u_{1}, \ldots\right)$ gives a system of congruence intersection terms.

For the converse, it is illuminating to work with maps on congruence lattices induced by surjections of algebras. Observe that if such maps in a variety do preserve finite meets then the variety must be congruence distributive. Indeed, for a surjection $\phi: \mathbf{A} \rightarrow \mathbf{B}$, the map $\phi^{*}: \operatorname{Con}(\mathbf{A}) \rightarrow \operatorname{Con}(\mathbf{B})$ is equivalent under the correspondence theorem to the map $\theta \mapsto \theta \vee \operatorname{ker} \phi$ of $\operatorname{Con}(\mathbf{A})$ to itself, for which preservation of meets gives the distributivity of joins with $\operatorname{ker} \phi$. Here $\operatorname{ker} \phi$ could be any congruence.

Given (2), then, and a surjection $\phi: \mathbf{A} \rightarrow \mathbf{B}$, observe that by the preservation properties of $\phi^{*}$ and the definition of a system of congruence terms, $\phi^{*}$ at least preserves the intersection of two principal congruences. We need to show that $\phi^{*}$ preserves the intersection of two arbitrary congruences, $\theta_{1}$ and $\theta_{2}$. Suppose by way of contradiction that

$$
\phi^{*}\left(\theta_{1} \cap \theta_{2}\right)<\phi^{*}\left(\theta_{1}\right) \cap \phi^{*}\left(\theta_{2}\right) .
$$

Extend the left-hand side of equation (2.6) to a completely meet-irreducible congruence $\psi$ on $\mathbf{B}$ not containing the right-hand side. Factoring out $\psi$ in the inequality (2.6) amounts to a reduction to the case where $B$ is subdirectly irreducible and the left-hand side of equation (2.6) is $0_{\mathbf{B}}$. Then $\phi^{*}\left(\theta_{1}\right)$ and $\phi^{*}\left(\theta_{2}\right)$ both contain the monolith of $\mathbf{B}$, a fact that remains true if $\theta_{1}$ and $\theta_{2}$ are replaced by a suitable principal congruence under each. But then we have a contradiction of the observation above that $\phi^{*}$ preserves the intersection of two principal congruences.

A system of congruence intersection terms without parameters is simply a system with parameters in which the terms do not actually use the parameters:

$$
\vartheta(a, b) \cap \vartheta(c, d)=\bigvee_{i \in I} \vartheta\left(p_{i}(a, b, c, d), q_{i}(a, b, c, d)\right)
$$

By Theorem 2.4 varieties with such a system are necessarily congruence distributive (as shown directly in [9]). These varieties are characterized as follows.

THEOREM 2.5. For a congruence distributive variety $\mathcal{V}$, the following conditions are equivalent:

(1) $V$ has a system of congruence intersection terms without parameters.

(2) There is a cardinal $\kappa$ such that in algebras of $\mathcal{V}$, the intersection of two principal congruences is the join of at most $\kappa$ principal congruences.

(3) Any nontrivial subalgebra of a finitely subdirectly irreducible algebra in $\mathcal{V}$ is finitely subdirectly irreducible. 
ProOF. That (1) implies (2) is immediate, taking $\kappa=|I|$. To verify that (2) implies (1), choose an infinite cardinal $\kappa^{\prime}>\kappa$ and in the free algebra $\mathbf{F}\left(x, y, z, w,\left\{u_{j}, j<\right.\right.$ $\left.\left.\boldsymbol{\kappa}^{\prime}\right\}\right)$ write $\vartheta(x, y) \cap \vartheta(z, w)$ as a join of principal congruences,

$$
\vartheta(x, y) \cap \vartheta(z, w)=\bigvee_{i \in I} \vartheta\left(p_{i}, q_{i}\right)
$$

where $p_{i}, q_{i}$ are terms evaluated on the generators and $|I| \leq \kappa$. Thus infinitely many generators $u_{j}$ are not involved in any $p_{i}$ or $q_{i}$ and so there exists a surjection $\sigma: \mathbf{F}\left(x, y, z, w,\left\{u_{j}, j<\kappa^{\prime}\right\}\right) \rightarrow \mathbf{F}\left(x, y, z, w,\left\{u_{j}, j<\omega\right\}\right)$ taking $x, y, z, w$ to themselves, all $u_{j}$ involved in some $p_{i}$ or $q_{i}$ to $x$, and each remaining $u_{j}$ to some $u_{j}$, with $j^{\prime}<\omega$. The images $\sigma\left(p_{i}\right), \sigma\left(q_{i}\right), i \in I$ satisfy the hypotheses of Lemma 2.3 and so form a system of congruence intersection terms, without involving any parameters.

To show that (1) implies (3), observe that by Corollary 2.2 , finitely subdirectly irreducible algebras are characterized by an infinitary universal sentence, namely

$$
\forall x y z w\left[\bigwedge_{i \in I}\left(p_{i}(x, y, z, w) \approx q_{i}(x, y, z, w)\right) \rightarrow(x \approx y) \vee(z \approx w)\right] .
$$

To show that (3) implies (1), take any system of congruence intersection terms with parameters (which exists by Theorem 2.4). Replacing the parameters by terms in $x, y, z, w$ in all possible ways, we obtain a new system of pairs of terms in $x, y, z, w$ alone. Now checking (2.2) for this new system reduces to checking (2.2) for the old system in the subalgebra B generated by $a, b, c, d$. But (2.2) holds in B by Corollary 2.2 , since $\mathbf{B}$ is finitely subdirectly irreducible. Now Lemma 2.1 yields the thesis.

We say that a variety has the $\operatorname{CIP}_{n}$ if it has a finite system of congruence intersection terms without parameters $\left\{\left(p_{i}, q_{i}\right): i=1, \ldots, n\right\}$. Any such variety, is congruence distributive and has the CIP (just use distributivity). On the other hand, any congruence distributive variety with the CIP must have a system of congruence intersection terms without parameters (take $\kappa=\omega$ in Theorem 2.5). In actuality something even stronger holds:

COROLLARY 2.6. Let $\mathcal{V}$ be a congruence distributive variety with the CIP. Then any system of congruence intersection terms without parameters can be reduced to a finite one.

PROOF. Let $\left\{\left(p_{i}, q_{i}\right): i \in I\right\}$ be a system of congruence intersection terms without parameters for $\mathcal{V}$. In the free algebra $\mathbf{F}=\mathbf{F}\left(x, y, z, w, u_{0}, u_{1}, \ldots\right)$ we have

$$
\vartheta_{\mathbf{F}}(x, y) \cap \vartheta_{\mathbf{F}}(z, w)=\bigvee_{i \in I} \vartheta_{\mathbf{F}}\left(p_{i}(x, y, z, w), q_{i}(x, y, z, w)\right)
$$


Since $\mathcal{V}$ has the CIP there must be $i_{1}, \ldots, i_{n} \in I$ such that

$$
\vartheta_{\mathbf{F}}(x, y) \cap \vartheta_{\mathbf{F}}(z, w)=\bigvee_{j=1, \ldots, n} \vartheta_{\mathbf{F}}\left(p_{i_{j}}(x, y, z, w), q_{i_{j}}(x, y, z, w)\right)
$$

From Lemma 2.3 , it follows that $\left\{\left(p_{i_{j}}, q_{i_{j}}\right): j=1, \ldots, n\right\}$ is a finite system of congruence intersection terms without parameters for $\mathcal{V}$.

COROLlaRY 2.7. (See also $[4,9]$ ) For any nontrivial variety $v$ the following are equivalent.

(1) $\mathcal{V}$ is congruence distributive and has the CIP.

(2) $\mathcal{V}$ has the $\mathrm{CIP}_{n}$ for some $n$.

In particular, a congruence distributive variety $\mathcal{V}$ has the PIP if and only if there are 4-ary terms $p, q$ such that for any $\mathbf{A} \in \mathcal{V}$ and $a, b, c, d \in A$

$$
\vartheta_{\mathbf{A}}(a, b) \cap \vartheta_{\mathbf{A}}(c, d)=\vartheta_{\mathbf{A}}(p(a, b, c, d), q(a, b, c, d))
$$

COROLLARY 2.8. ([4, Theorem 2.15]) If $\mathcal{V}$ has the $\mathrm{CIP}_{n}$, then the class of finitely subdirectly irreducible algebras in $\mathcal{V}$ is strictly elementary, relative to $\mathcal{V}$.

EXAMPLE 2.9. Let $\mathcal{V}$ be the variety generated by a finite nondistributive lattice, say the pentagon $\mathbf{N}_{5}$. By the construction in Section 3 of [4], $\mathcal{V}$ has a finite system of congruence intersection terms with parameters. We cannot dispose of the parameters by Theorem 2.5: the three-element chain is subalgebra of $\mathbf{N}_{5}$, but it is not finitely subdirectly irreducible.

EXAMPLE 2.10. The construction in Section 3 of [4] shows that the variety $\mathcal{L}$ of all lattices has an infinite set of congruence intersection terms with parameters. Such a system can be reduced neither to an infinite set without parameters, nor to a finite set with parameters.

EXAMPLE 2.11. Filtral varieties were introduced by Magari [16]; the most accessible reference is [17] but see also $[7,8,10,11]$. A variety $\mathcal{V}$ is filtral if every congruence on a subdirect product $\mathbf{A} \subseteq \Pi_{i \in I} \mathbf{B}_{i}$ of subdirectly irreducible algebras $\mathbf{B}_{i} \in \mathcal{V}, i \in I$ is of the form $\theta_{F}$, where $F$ is a filter on $I$ and $(a, b) \in \theta_{F}$ if and only if $\left\{i \in I: a_{i}=b_{i}\right\} \in F$. A nontrivial variety $\mathcal{V}$ is filtral if and only if the compact congruences on each algebra in $\mathcal{V}$ form a dual generalized Boolean algebra [7]. It follows immediately that any filtral variety has the CIP. Examples include distributive lattices, Boolean algebras, and some other varieties from algebraic logic. Filtral varieties will be used further in Section 4. 


\section{Non-congruence-distributive varieties}

In the absence of congruence distributivity, the situation is much more complicated. By Corollary 2.7 above the CIP and the PIP are not expressible via terms, nor can we affirm that the PIP implies the CIP.

Therefore verifying the CIP or the PIP for a non-congruence-distributive variety can be difficult. Fortunately there is a 'lifting' theorem for reducts that applies to any variety $\mathcal{V}$, congruence distributive or not. Let $F \subseteq \operatorname{Clo}(\mathcal{V})$. Let $\operatorname{Rd}_{F}(\mathbf{A})$ denote the $F$-reduct of $\mathbf{A}$ for $\mathbf{A} \in \mathcal{V}$ and let $\operatorname{Rd}_{F}(\mathcal{V})$ denote the variety generated by the $F$-reducts of algebras in $\mathcal{V}$.

THEOREM 3.1. Let $\mathcal{V}$ be any variety. Suppose that

(1) for some $F \subseteq \operatorname{Clo}(\mathcal{V}), \operatorname{Rd}_{F}(\mathcal{V})$ has the $\mathrm{CIP}$;

(2) any principal congruence of any $A \in \mathcal{V}$ is compact in $\operatorname{Con}\left(\operatorname{Rd}_{F}(A)\right)$.

Then $\mathcal{V}$ has the CIP.

PROOF. A compact congruence $\alpha$ on $A \in \mathcal{V}$ remains compact in $\operatorname{Con}\left(\operatorname{Rd}_{F}(A)\right)$, since $\alpha$ is the join of finitely many principal congruences of $\mathbf{A}$, each of which is compact in $\operatorname{Con}\left(\operatorname{Rd}_{F}(A)\right)$. For compact $\alpha, \beta \in \operatorname{Con}(A), \alpha \cap \beta$ is then compact in $\operatorname{Con}\left(\operatorname{Rd}_{F}(\mathbf{A})\right)$ by hypothesis and hence is compact in $\operatorname{Con}(\mathbf{A})$, because a compact element in a lattice remains compact in any complete sublattice containing it.

One particular instance of Theorem 3.1 is very useful. Let $\mathcal{V}$ be a variety and let $\mathbf{S}$ be a semigroup acting endomorphically on the elements of $\mathcal{V}$. In other words, for each $\mathbf{A} \in \mathcal{V}$ and $\sigma \in S$ there is an endomorphism of $\mathbf{A}$, also called $\sigma$, such that for any $\sigma, \tau \in S$ and $a \in A$

$$
\sigma(\tau(a))=(\sigma \tau)(a)
$$

For any $\mathbf{A}=\langle A, F\rangle \in \mathcal{V}$ let $\mathbf{A}[\mathbf{S}]=\langle A, F, S\rangle \in \mathcal{V}[\mathbf{S}]$ be the corresponding algebra in which the endomorphisms in the action of $\mathbf{S}$ are considered additional operations. Let $\mathcal{V}[\mathbf{S}]$ be the variety generated by the algebras $\mathbf{A}[\mathbf{S}]$ for $\mathbf{A} \in \mathcal{V}$. The endomorphic action of $\mathbf{S}$ on the members of $\mathcal{V}$ is expressible by laws of $\mathcal{V}[\mathbf{S}]$.

LEMMA 3.2. Let $\mathcal{V}$ be a variety and let $\mathbf{S}$ be any semigroup acting endomorphically on $\mathcal{V}$. Then for any $\mathbf{A} \in \mathcal{V}$ and any $a, b \in A$

$$
\vartheta_{\mathbf{A}[\mathbf{S}]}(a, b)=\bigvee_{\sigma \in S} \vartheta_{\mathbf{A}}(\sigma(a), \sigma(b))
$$

PROOF. We may assume without loss of generality that $\mathbf{S}$ has an identity element. One of the many versions of the Mal'tsev construction of principal congruences is as 
follows. Starting with $(a, b)$ form additional pairs by repeatedly applying the unary polynomials that are obtained from the basic operations by fixing all variables except for one. The equivalence closure of such a set of pairs is the principal congruence generated by $(a, b)$.

Observe that in our case a composition of endomorphisms following a unary polynomial can be rewritten as a unary polynomial following the endomorphisms; moreover, since $\mathbf{S}$ is closed under composition, it is sufficient to apply just one endomorphism preceding unary polynomials. In other words, to compute $\vartheta_{\mathbf{A}[\mathbf{S}\}}(a, b)$, one can start from $(a, b)$, then apply an endomorphism, then the unary polynomials necessary for computing $\vartheta_{\mathbf{A}}(a, b)$ and finally take the transitive closure. But this is precisely what (3.1) asserts.

A straightforward application of Theorem 3.1 yields the following fact.

PROPOSITION 3.3. Let $\mathcal{V}$ be a variety and let $\mathbf{S}$ be a finite semigroup acting endomorphically on $\mathcal{V}$. If $\mathcal{V}$ has the CIP, so does $\mathcal{V}[\mathrm{S}]$.

COROLLARY 3.4. Let $\mathbf{A}=\left\langle A, f_{1}, \ldots, f_{k}\right\rangle$ be a finite algebra generating a variety with the CIP. Let $\sigma_{1}, \ldots, \sigma_{n}$ be endomorphisms of $\mathbf{A}$ and let $\mathbf{A}^{\prime}=\left\langle A, f_{1}, \ldots, f_{k}\right.$, $\left.\sigma_{1}, \ldots, \sigma_{n}\right)$. Then $\mathrm{V}\left(\mathbf{A}^{\prime}\right)$ has the CIP.

PROOF. Since $\mathbf{A}$ is finite the endomorphism monoid $\mathbf{S}$ generated by $\sigma_{1}, \ldots, \sigma_{n}$ and the identity function is finite. Then $\mathbb{V}(\mathbf{A}[\mathbf{S}])$ has the CIP, via Proposition 3.3. But clearly a member of $\mathbb{V}\left(\mathbf{A}^{\prime}\right)$ has the same congruence lattice as the corresponding member of $\mathbb{V}(\mathbf{A}[\mathbf{S}])$.

Let us apply these results to unary varieties (varieties in which every basic operation has arity at most 1). First, observe that the variety of sets with no operations has the PIP and CIP, since compact equivalence relations in a partition lattice form an ideal and principal equivalence relations are atoms.

Since an endomorphism of a set is just a unary function, any variety $\mathcal{V}$ of unary algebras can be viewed as $\mathcal{S}[\mathbf{M}]$, where $S$ is the variety of sets and $\mathbf{M}=\langle\mathrm{Clo}(\mathcal{V}), 0\rangle$. Since the variety of sets has the CIP, from Proposition 3.3 and Corollary 3.4 we get at once.

PROPOSITION 3.5. If $\mathcal{V}$ is a variety of unary algebras and $\operatorname{Clo}(\mathcal{V})$ is finite, then $\mathcal{V}$ has the CIP. Any finitely generated variety of unary algebras has the CIP.

Actually, something more can be proved with a slightly different argument.

PROPOSITION 3.6. Any locally finite variety of unary algebras has the CIP. 
Proof. Let $\mathcal{V}$ be locally finite and let $\mathbf{A} \in \mathcal{V}$. Any compact congruence $\theta$ of $\mathbf{A}$ is generated by finitely many pairs of elements. Since $\mathbf{A}$ is unary the only nonsingleton blocks of $\theta$ are contained in the subalgebra generated by those elements. This subalgebra must be finite since $\mathcal{V}$ is locally finite. Hence a congruence of $\mathbf{A}$ is compact if and only if it has finitely many nontrivial blocks, all finite. Thus the compact congruences of $\mathbf{A}$ form an ideal in the congruence lattice of $\mathbf{A}$ and so are closed under intersection.

In general varieties of unary algebras do not have the PIP. Choose any algebra $\mathbf{A}$ without the PIP and consider the algebra $\mathbf{B}=\left\langle A, \operatorname{Pol}_{1}(\mathbf{A})\right\rangle$. Then the variety $\mathcal{V}=\mathbb{V}(\mathbf{B})$ will not have the PIP and, if $\mathbf{A}$ is finite, $\mathcal{V}$ will be locally finite.

For varieties of monounary algebras (just one unary operation $f$ ) a full characterization of the PIP can be given. First, two key examples.

EXAMPLE 3.7. If a monounary algebra $\mathbf{A}$ is 1 -generated, then all congruence relations on $\mathbf{A}$ are principal, so that $\mathbf{A}$ has the PIP.

EXAMPLE 3.8. Let $\mathbf{A}_{m, n}=\mathbf{C}_{m} \dot{\cup} \mathbf{C}_{n}$, the disjoint union of an $m$-cycle and an $n$-cycle as monounary algebras. If $1<\operatorname{gcd}(m, n)<m, n$, then $\mathbf{A}$ does not have the PIP. Indeed, if $a$ generates $\mathbf{C}_{m}$ and $b$ generates $\mathbf{C}_{n}$, then $\vartheta_{\mathbf{A}}(a, b) \cap \vartheta_{\mathbf{A}}(a, f(b))$ is not principal.

PROPOSITION 3.9. For a monounary variety $\mathcal{V}$ the following are equivalent.

(1) $V$ has the PIP;

(2) $\mathbf{A}_{m, n} \notin \mathcal{V}$ for $1<\operatorname{gcd}(m, n)<m, n$;

(3) $\mathcal{V}$ satisfies the law $f^{k+h}(x) \approx f^{k}(x)$, for some $k \geq 0$ and $h \geq 1$, where $h$ is either a power of a prime or the product of two distinct primes.

Proof. (Outline.) Example 3.8 shows that (1) implies (2). It is also evident that (3) implies (2), since in $\mathbf{A}_{m, n}$ with $1<\operatorname{gcd}(m, n)<\boldsymbol{m}, n$ any law of the form in (3) fails. That (2) implies (3) follows in the contrapositive from representing $\mathbf{A}_{m, n}$ as a homomorphic image of $\mathbf{F}_{\mathcal{V}}(2)=\mathbf{F}_{\mathcal{V}}(1) \dot{\cup} \mathbf{F}_{\mathcal{V}}(1)$ (unless $\mathcal{V}$ satisfies a law $f^{i}(x) \approx f^{j}(y)$, a case handled separately). Finally, the implication from (2) to (1) is best approached through the lemma that the restriction of a principal congruence to a subalgebra is again principal; in considering an intersection $\vartheta_{\mathbf{A}}(a, b) \cap \vartheta_{\mathbf{A}}(c, d)$ one can then assume that $\{a, b\}$ and $\{c, d\}$ generate the same subalgebra, thus leading to examples of the form $\mathbf{A}_{m, n}$ of which (2) excludes those lacking the PIP.

\section{Applications to congruence distributive varieties}

For congruence distributive varieties, we can refine the results of Section 3, for two reasons. The first is that Lemma 3.2 will hold for any semigroup of endomorphisms, 
finite or not. The second is purely lattice-theoretic; congruence lattices in such varieties are distributive algebraic lattices and so satisfy the join infinite distributive identity (JID). For any $a \in L$ and for any family $\left(b_{i}\right)_{i \in I} \subseteq L$

$$
a \wedge \bigvee_{i \in I} b_{i}=\bigvee_{i \in I}\left(a \wedge b_{i}\right)
$$

THEOREM 4.1. Let $\mathcal{V}$ be a congruence distributive variety and let $\mathbf{S}$ be a semigroup acting endomorphically on $\mathcal{V}$. If $p_{i}(x, y, z, w), q_{i}(x, y, z, w), i \in I$, is a system of congruence intersection terms without parameters for $\mathcal{V}$, then

$$
p_{i}(x, y, \sigma(z), \sigma(w)), q_{i}(x, y, \sigma(z), \sigma(w)), \quad i \in I, \sigma \in S,
$$

form a system of congruence intersection terms without parameters for $\mathcal{V}[\mathbf{S}]$.

PROOF. Note that, since we can add an identity to $\mathbf{S}$, with no loss of generality we may safely assume that $S^{2}=S$.

If $\mathbf{A}^{\prime}=\mathbf{A}[\mathbf{S}] \in \mathcal{V}[\mathbf{S}]$ and $a, b, c, d \in A$, then by Lemma 3.2 and by the JID (applied twice)

$$
\begin{aligned}
\vartheta_{\mathbf{A}^{\prime}}(a, b) & \cap \vartheta_{\mathbf{A}^{\prime}}(c, d) \\
= & \bigvee_{\sigma \in S} \vartheta_{\mathbf{A}}(\sigma(a), \sigma(b)) \cap \bigvee_{\tau \in S} \vartheta_{\mathbf{A}}(\tau(c), \tau(d)) \\
= & \bigvee_{\sigma, \tau \in S}\left(\vartheta_{\mathbf{A}}(\sigma(a), \sigma(b)) \cap \vartheta_{\mathbf{A}}(\tau(c), \tau(d))\right) \\
= & \bigvee_{\sigma, \tau \in S} \bigvee_{i \in I} \vartheta_{\mathbf{A}}\left(p_{i}(\sigma(a), \sigma(b), \tau(c), \tau(d)), q_{i}(\sigma(a), g m a(b), \tau(c), \tau(d))\right)
\end{aligned}
$$

On the other hand, again by Lemma 3.2,

$$
\begin{aligned}
\bigvee_{\tau \in S} \bigvee_{i \in I} \vartheta_{\mathrm{A}[\mathbf{S}]}\left(p_{i}(a, b, \tau(c), \tau(d)), q_{i}(a, b, \tau(c), \tau(d))\right) \\
\quad=\bigvee_{\tau, \sigma \in S} \bigvee_{i \in I} \vartheta_{\mathbf{A}}\left(\sigma\left[p_{i}(a, b, \tau(c), \tau(d))\right], \sigma\left[q_{i}(a, b, \tau(c), \tau(d))\right]\right) \\
=\bigvee_{\tau, \sigma \in S} \bigvee_{i \in I} \vartheta_{\mathbf{A}}\left(p_{i}(\sigma(a), \sigma(b), \sigma \tau(c), \sigma \tau(d)), q_{i}(\sigma(a), \sigma(b), \sigma \tau(c), \sigma \tau(d))\right) \\
\quad=\bigvee_{\sigma, \tau \in S} \bigvee_{i \in I} \vartheta_{\mathbf{A}}\left(p_{i}(\sigma(a), \sigma(b), \tau(c), \tau(d)), q_{i}(\sigma(a), \sigma(b), \tau(c), \tau(d))\right)
\end{aligned}
$$

where the last equality holds since $S^{2}=S$. We have thus shown that the chosen terms do form a system of congruence intersection terms for $\mathcal{V}[\mathbf{S}]$. 
From Theorem 4.1 it is clear that, if we start from a congruence distributive variety $\mathcal{V}$ with the $\mathrm{CIP}_{n}$ and $\mathbf{S}$ is finite, then the $\mathrm{CIP}_{m}$ holds for $\mathcal{V}[\mathbf{S}]$ for some $m$. To what extent can we determine $m$ ? It is clear that the cardinality of $\mathbf{S}$ plays an important role, while the constant endomorphisms do not contribute.

PROPOSITION 4.2. Suppose that $\mathcal{V}$ is a congruence distributive variety with the $\mathrm{CIP}_{n}$. Let $\hat{\mathbf{S}}$ be a finite semigroup acting endomorphically on $\mathcal{V}$ and suppose that $\mid\{\sigma \in \hat{S}: \sigma$ is not constant $\} \mid=k$. Then $\mathcal{V}[\hat{\mathbf{S}}]$ has the $\mathrm{CIP}_{m}$ for $m=k n$.

Our results can be applied to those varieties dubbed $M$-BD by Cornish in [14] and to their subvarieties. The algebras of $M$-BD are bounded distributive lattices with a semigroup $\mathbf{S}$ of endomorphisms or dual endomorphisms acting on them. If $\mathcal{D}$ is the variety of distributive lattices, then in our notation these varieties are $\mathcal{D}[S]$ for some S. If we take $\mathbf{S}$ to be the absolutely free monoid on one generator, then we get the variety of Ockham algebras, which is a good source of examples. In particular such a variety has an infinite system of congruence intersection terms without parameters for which no finite system can be found.

EXAMPLE 4.3. An Ockham algebra is an algebra $\langle A, \vee, \wedge, f, 0,1\rangle$, where $\langle A, \vee, \wedge, 0,1\rangle$ is a bounded distributive lattice and $f$ is a dual lattice endomorphism: for any $a, b \in A$

$$
f(a \vee b)=f(a) \wedge f(b) \quad f(a \wedge b)=f(a) \vee f(b) \quad f(0)=1 \quad f(1)=0 .
$$

Let $\mathcal{K}$ be the variety of Ockham algebras and let $\mathcal{K}_{p, q}(p \geq 1, q \geq 0)$ be the variety of Ockham algebras satisfying

$$
f^{q}(x) \approx f^{2 p+q}(x)
$$

The varieties $\mathcal{K}_{\text {and }} \mathcal{K}_{p, q}$ were introduced by Berman in [6]. Such varieties encompass many previously studied varieties; for instance the subvariety of $\mathcal{X}_{1,0}$ given by the equation $x \wedge f(x) \approx 0$ is the variety of Boolean algebras. Boolean algebras give evidence that Proposition 4.2 does not exclude having $\mathcal{V}[\mathbf{S}]$ satisfy the $\mathrm{CIP}_{\boldsymbol{m}^{\prime}}$ for some $m^{\prime}<m$. Distributive lattices have a majority operation $m(x, y, z)$ and the unique subdirectly irreducible algebra is the two-element chain. Given that, it is easily checked that the variety of distributive lattices has the PIP, witness $p(x, y, z, w)=$ $m(x, y, z)$ and $q(x, y, z, w)=m(x, y, w)$. It follows that Boolean algebras have at worst the $\mathrm{CIP}_{2}$. However Boolean algebras form a discriminator variety, so in fact they have the PIP (see for instance [12]).

Next the subvariety of $\mathcal{K}_{1,1}$ likewise determined by $x \wedge f(x) \approx 0$ is the variety of Stone algebras, and finally the variety $\mathcal{K}_{1,0}$ is the variety of de Morgan algebras. This is a very interesting variety which will be discussed at length in [2]. For more 
information about varieties of Ockham algebras we direct the reader to [1] and to the extended bibliography therein.

To construct an infinite system of congruence intersection terms for $\mathcal{K}$ itself, observe that by Lemma 3.2 (but see also [6, Theorem 1]), if $\mathbf{A} \in \mathcal{K}$ and $a, b \in A$

$$
\vartheta_{\mathbf{A}}(a, b)=\bigvee_{n \in \omega} \vartheta_{\mathbf{A}_{d}}\left(f^{n}(a), f^{n}(b)\right),
$$

where $\mathbf{A}_{d}$ is the underlying distributive lattice. Hence, if $m(x, y, z)$ is the usual majority term for distributive lattices, a set of congruence intersection terms without parameters for $\mathcal{K}$ is

$$
m\left(x, y, f^{n}(z)\right), \quad m\left(x, y, f^{n}(w)\right), \quad n \in \omega .
$$

(Note that from Proposition 4.2 it follows that each variety $\mathcal{K}_{p, q}$ has the $\mathrm{CIP}_{n}$ for $n=2 p+q-1$.)

To prove that no finite system of congruence intersection terms without parameters can be found for $\mathcal{K}$ it would be enough to show that $\mathcal{K}$ does not have the CIP, hence to display an Ockham algebra in which the meet of two principal congruences is not compact. However since the varieties $\mathcal{K}_{p, q}$ and their subvarieties coincide with varieties of finite height (see [1]) such an example might be hard to construct. Thus we adopt indirect reasoning, based on Corollary 2.6.

If $\mathcal{K}$ had the CIP, then by Corollary 2.6 the system $m\left(x, y, f^{n}(z)\right), m\left(x, y, f^{n}(w)\right)$, $n \in \omega$ could be reduced to a finite one. Hence there would be a $k$ such that for all $\mathbf{A} \in \mathcal{K}$ and $a, b, c, d \in A$

$$
\vartheta_{\mathbf{A}}(a, b) \cap \vartheta_{\mathbf{A}}(c, d)=\bigvee_{0 \leq n \leq k} \vartheta_{\mathbf{A}}\left(m\left(a, b, f^{n}(c)\right), m\left(a, b, f^{n}(d)\right)\right)
$$

We shall produce an algebra $\mathbf{L} \in \mathcal{K}$ and elements of $\mathbf{L}$ for which no such $k$ can exist.

Let $L=\{0,1\} \cup\left\{a_{n}: n \in \mathbb{Z}\right\}$ considered as a 0,1 chain. In other words 0 is the smallest element, 1 the largest, and $a_{n}<a_{m}$ if and only if $n<m$ in $\mathbb{Z}$. Define a unary operation $f$ on $L$ by $f(0)=1, f(1)=0, f\left(a_{0}\right)=a_{0}, f\left(a_{n}\right)=a_{-(n-1)}$ if $n>0$ and $f\left(a_{n}\right)=a_{-n}$ if $n<0$. The following facts are easily checked.

- $\mathbf{L}=\langle L, \vee, \wedge, f, 0,1\rangle$ is an Ockham algebra.

- $\operatorname{Con}(\mathbf{L})$ is an ordered chain isomorphic to $\omega+2$ and hence $\mathbf{L}$ is subdirectly irreducible.

- The monolith of $\mathbf{L}$ is $\vartheta_{\mathbf{L}}\left(a_{0}, a_{1}\right)$, which has a unique nontrivial block consisting exactly of $a_{0}$ and $a_{1}$.

- Any compact congruence of $\mathbf{L}$ collapses only finitely many elements except for the total congruence $\vartheta_{\mathbf{L}}(0,1)$. It follows that in $\mathbf{L}$ the intersection of two compact congruences is compact. 
Now, for any $c \neq d$ in $L$ with $c<d$, we must have

$$
\begin{aligned}
\vartheta_{\mathbf{L}}\left(a_{0}, a_{1}\right) & =\vartheta_{\mathbf{L}}\left(a_{0}, a_{1}\right) \cap \vartheta_{\mathbf{L}}(c, d)=\bigvee_{n \in \omega} \vartheta_{\mathbf{A}}\left(m\left(a_{0}, a_{1}, f^{n}(c)\right), m\left(a_{0}, a_{1}, f^{n}(d)\right)\right) \\
& =\bigvee_{n \in \omega} \vartheta_{\mathbf{A}}\left(a_{1} \wedge\left(a_{0} \vee f^{n}(c)\right), a_{1} \wedge\left(a_{0} \vee f^{n}(d)\right)\right) .
\end{aligned}
$$

Since $\vartheta_{\mathbf{A}}\left(a_{0}, a_{1}\right)$ is completely join irreducible,

$$
\vartheta_{\mathbf{A}}\left(a_{0}, a_{1}\right)=\vartheta_{\mathbf{A}}\left(a_{1} \wedge\left(a_{0} \vee f^{\bar{n}}(c)\right), a_{1} \wedge\left(a_{0} \vee f^{\bar{n}}(d)\right)\right)
$$

for some $\bar{n}$ and hence $\left\{a_{0}, a_{1}\right\}=\left\{a_{1} \wedge\left(a_{0} \vee f^{\bar{n}}(c)\right), a_{1} \wedge\left(a_{0} \vee f^{\bar{n}}(d)\right)\right\}$.

Next observe that, in $L$, if $x, y \geq a_{1}$ or $x, y \leq a_{0}$ then

$$
\left\{a_{0}, a_{1}\right\} \neq\left\{a_{1} \wedge\left(a_{0} \vee x\right), a_{1} \wedge\left(a_{0} \vee y\right)\right\}
$$

If $\mathcal{K}$ had the CIP, say the $\operatorname{CIP}_{k}$ for some $k$, then such an $\bar{n}$ would lie between 0 and $k$. But for any choice of $k \geq 1$ take $c=a_{k}$ and $d=a_{k+1}$ in $L$; then

$$
\begin{aligned}
& a_{k}, a_{k+1} \geq a_{1} \\
& f\left(a_{k}\right), f\left(a_{k+1}\right) \leq a_{0} \\
& f^{2}\left(a_{k}\right), f^{2}\left(a_{k+1}\right) \geq a_{1} \\
& \ldots \\
& f^{k}\left(a_{k}\right), f^{k}\left(a_{k+1}\right) \leq a_{0} \quad \text { if } k \text { is odd } \\
& f^{k}\left(a_{k}\right), f^{k}\left(a_{k+1}\right) \geq a_{1} \quad \text { if } k \text { is even. }
\end{aligned}
$$

This shows that no such $k$ can exist and hence $\mathcal{K}$ does not have the CIP.

We are now able to give an example to show that the PIP for an algebra does not depend solely on the isomorphism type of the congruence lattice. This example illustrates a failure of the PIP in an explicit way; however the algebra and its variety do have the CIP. The construction is based on the observation that, in a chain, collapsing an interval $[a, b]$ gives a principal congruence relation but collapsing two disjoint intervals simultaneously does not.

EXAMPLE 4.4. Let $\mathbf{B}=\langle\{0,1,2,3,4\}, \vee, \wedge\rangle$ regarded as a finite distributive lattice. Let $\sigma_{1}, \sigma_{2}$ be the endomorphisms described by the following tables:

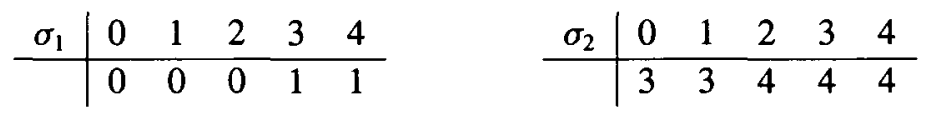


Any nontrivial distributive lattice generates the variety of distributive lattices, so Corollary 3.4 applies to show that the algebra $\mathbf{B}^{\prime}=\left\langle\{0,1,2,3,4\}, \vee, \wedge, \sigma_{1}, \sigma_{2}\right\rangle$ generates a variety with the CIP. This variety does not have the PIP, though, since $\mathbf{B}^{\prime}$ does not; in $\operatorname{Con}\left(\mathbf{B}^{\prime}\right)$ as shown in Figure 2, the element $\varphi=\vartheta_{\mathbf{B}^{\prime}}(0,2) \cap \vartheta_{\mathbf{B}^{\prime}}(2,4)=$ $\vartheta_{\mathbf{B}^{\prime}}(3,4) \vee \vartheta_{\mathbf{B}^{\prime}}(0,1)$ is not principal. Moreover, since both $\sigma_{1} \circ \sigma_{2}$ and $\sigma_{2} \circ \sigma_{1}$ are constant we can conclude by Proposition 4.2 that $\mathbb{V}\left(\mathbf{B}^{\prime}\right)$ has the $\mathrm{CIP}_{3}$.

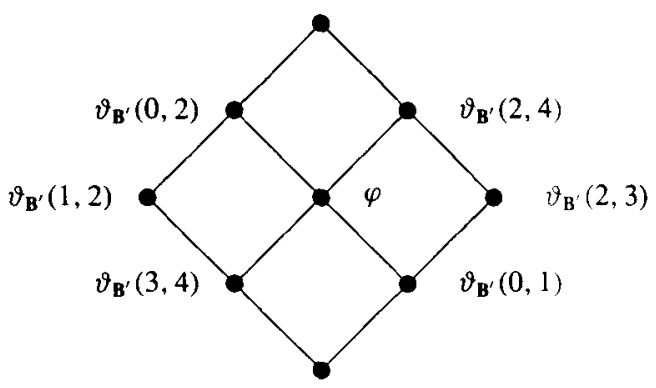

FIGURE 2. The congruence lattice of $\mathbf{B}^{\prime}$

Consider $\mathbf{A}=\mathbf{B}^{\prime} / \vartheta_{\mathbf{B}^{\prime}}(1,2)$ and let $\mathbf{C}=\mathbf{A} \times \mathbf{A}$. Then $\operatorname{Con}(\mathbf{A})$ is the threeelement chain (by Figure 2) and $\mathbf{A}$ has the PIP. Since $\mathbf{A}$ generates a congruence distributive variety the only congruences of $\mathbf{C}$ are product congruences and hence $\operatorname{Con}(\mathbf{C}) \cong \operatorname{Con}(\mathbf{B})^{\prime}$. Note that $\mathbf{C}$ has the PIP, while $\mathbf{B}^{\prime}$ does not.

Next, for each $n$ we shall construct a congruence distributive variety having the CIP (and hence the $\mathrm{CIP}_{k}$ for some $k$ ) but not the $\mathrm{CIP}_{n}$ (Example 4.6).

Let $X$ be a set. By the pointed majority algebra based on $X$, let us mean the algebra $\widehat{\mathbf{X}}=\langle X \cup\{0\}, m, 0\rangle$ where $0 \notin X$ and $m$ is the ternary operation such that, for any $u, v, w \in X \cup\{0\}, m(u, v, w)=0$ if $u, v, w$ are distinct, and

$$
m(v, v, w)=m(v, w, v)=m(w, v, v)=v
$$

Hence $m(x, y, z)$ is a majority operation on $\mathbf{X}$ and so $\mathbf{V}(\widehat{\mathbf{X}})$ is congruence distributive. An equivalent construction is to form the cube $\{0,1\}^{X}$, to endow it with the unique majority operation $m$ and to take the subalgebra $\mathbf{Y}$ consisting of all vertices with at most one coordinate value of 1 . This subalgebra is an isomorphic copy of $\widehat{\mathbf{X}}$. It can be shown (see [3]), that $\langle\{0,1\}, m\rangle$ is filtral. This means that each congruence relation $\theta$ on a power is induced by a filter $\mathcal{F}$ on the set of indices: $(\mathbf{a}, \mathbf{b}) \in \theta$ if and only if $\left\{i \in X: a_{i}=b_{i}\right\} \in \mathcal{F}$. Since any two elements of $\mathbf{Y}$ disagree in at most two indices, $\theta$ is determined by the set of indices such that $\{i\} \notin \mathcal{F}$. In terms of the isomorphic copy $\widehat{\mathbf{X}}$, the congruence relation $\vartheta_{\widehat{\mathbf{x}}}(S)$ generated by the pairs belonging to $S \subseteq X$ identifies the elements of $S$ with 0 and leaves all other elements as singletons. Moreover, any 
congruence of $\widehat{\mathbf{X}}$ is of this form. It can be shown (see [3]) that the variety generated by $\langle\{0,1\}, m\rangle$ has the PIP.

Now let $G=\langle W, E, \varepsilon, 0\rangle$ be a pointed directed graph with vertex set $W$, edge set $E$, and edge map $\varepsilon$ (so that for $e \in E, \varepsilon(e)=(v, w)$ if $e$ is an edge from $v$ to $w$ ). Let 0 be a new element not in $W$. We define the corresponding majority graph algebra to be $\widehat{\mathbf{G}}=\left\langle W \cup\{0\}, m, 0, \sigma_{e}\right\rangle_{e \in E}$, where $\langle W \cup\{0\}, m, 0\rangle$ is a pointed majority algebra and for any $e \in E$ and $v \in W \cup\{0\}$

$$
\sigma_{e}(v)= \begin{cases}w & \text { if } \varepsilon(e)=(v, w) \\ 0 & \text { otherwise }\end{cases}
$$

The congruence lattice of a majority graph algebra is thus the sublattice of the congruence lattice of the corresponding pointed majority algebra consisting of those members compatible with the maps $\sigma_{e}$. This congruence lattice is easily described.

LEMMA 4.5. Let $\widehat{\mathbf{G}}=\left\langle W \cup\{0\}, m, 0, \sigma_{e}\right\rangle_{e \in E}$ be a majority graph algebra. For any $\alpha \subseteq W \times W$ the following are equivalent:

(1) $\alpha \in \operatorname{Con}(\widehat{\mathbf{G}})$;

(2) $\alpha=\vartheta_{\widehat{\mathbf{G}}}(S)$ for some $S \subseteq W$ which contains 0 and is edge closed, in the sense that if $v \in S$ and there is an edge from $v$ to $w$, then $w \in S$.

The map sending $S \mapsto \vartheta_{\widehat{\mathrm{G}}}(S)$ is a lattice isomorphism from the lattice of edge-closed subsets of $\widehat{\mathbf{G}}$ and $\operatorname{Con}(\widehat{\mathbf{G}})$. It follows that $\vartheta_{\widehat{\mathbf{G}}}(u, v)=\vartheta_{\widehat{\mathbf{G}}}(S)$, where $S$ consists of $u, v, 0$ and all the vertices reachable by these via a directed path.

EXAMPLE 4.6. Let $W_{n}=\left\{a, b, c_{1}, \ldots, c_{2 n+2}\right\}$ and let $G_{n}=\left\langle W_{n}, E_{n}, \varepsilon\right\rangle$ be the directed graph with an edge from $a$ to $c_{i}$ and from $b$ to $c_{i}$ for $i=1, \ldots, 2 n+2$ and no other edge. Consider the majority graph algebra $\widehat{\mathbf{G}}_{n}=\left\langle W_{n} \cup\{0\}, m, 0, \sigma_{e}\right\rangle_{e \in E_{n}}$. For any $e \in E_{n}, \sigma_{e}$ is an endomorphism of the underiying pointed majority algebra $\left\langle W_{n} \cup\right.$ $\{0\}, m, 0\rangle$. Since such endomorphisms generate a finite semigroup of endomorphisms and $\vee\left(\left\langle W_{n} \cup\{0\}, m, 0\right\rangle\right)$ has the PIP, $\vee\left(\widehat{\mathbf{G}}_{n}\right)$ has the CIP by Corollary 3.4.

We can be more precise. Since $\sigma_{e} \circ \sigma_{f}$ is the constant endomorphism with value 0 for any $e, f \in E_{n}$, it follows that there are only $2 n+3$ nonconstant endomorphisms and so, by Proposition 4.2, $\mathbb{V}\left(\widehat{\mathbf{G}}_{n}\right)$ has the $\operatorname{CIP}_{2 n+3}$.

$\vee\left(\widehat{\mathbf{G}}_{n}\right)$ does not have the $\operatorname{CIP}_{n}$ though: From $\vartheta_{\widehat{\mathbf{G}}_{n}}(0, a)=\vartheta_{\widehat{\mathbf{G}}_{n}}\left(\left\{0, a, c_{1}, \ldots, c_{2 n+2}\right\}\right)$ and $\vartheta_{\widehat{\mathbf{G}}_{n}}(0, b)=\vartheta_{\widehat{\mathbf{G}}_{n}}\left(\left\{0, b, c_{1}, \ldots, c_{2 n+2}\right\}\right)$, it follows that

$$
\vartheta_{\widehat{\mathbf{G}}_{n}}(0, a) \cap \vartheta_{\widehat{\mathbf{G}}_{n}}(0, b)=\bigvee_{k=1}^{2 n+1} \vartheta_{\widehat{\mathbf{G}}_{n}}\left(c_{k}, c_{k+1}\right)
$$

the latter is a join of $n+1$ congruence relations and, because no edges originate among the $c_{i}$, there is no way to express it as a join of fewer principal congruence relations. 
EXAMPLE 4.7. In the introduction it was claimed that the CIP is a purely latticetheoretic property while the PIP may depend on the operations. Example 4.4 displayed two algebras having isomorphic congruence lattices, one having the PIP and the other the CIP but not the PIP. We can now give an example of two varieties whose members have the same congruence lattices up to isomorphism, where one variety has the PIP and the other has the CIP but not the PIP.

Let $\mathcal{D}$ be the variety of distributive lattices, which has the PIP. Consider now the lattice term

$$
p(x, y, z)=x \vee(y \wedge z),
$$

let $2=\{0,1\}$ and let $\mathcal{P}=\mathbb{V}(\langle 2, p\rangle)$. In [3] it is shown that $\mathcal{P}$ is filtral. Moreover $\mathcal{P}$ does not have the PIP, for in the free algebra in $\mathcal{P}$ generated by $x, y, z$ the congruence $\vartheta(x, y) \cap \vartheta(y, z)$ is not principal. Given $\mathbf{A} \in \mathcal{P}$, we seek $\mathbf{L} \in \mathcal{D}$ with $\operatorname{Con}(\mathbf{L}) \cong$ Con(A). Since $\mathcal{P}$ is clearly semisimple and congruence distributive, $\mathbf{A}$ is isomorphic to a subdirect product of copies of $\langle 2, p\rangle$; in fact we may assume that $\mathbf{A}$ is such a subdirect product. Using lattice operations on 2 , let $\mathbf{L}$ be the sublattice generated by the elements of $\mathbf{A}$, within the same enveloping product. Since $p(x, y, y) \approx x \wedge y, \mathbf{A}$ is already closed under meets, so by distributivity $\mathbf{L}$ consists of finite joins of elements of A. Observe also that $\mathbf{A}$ has 'bounded joins', in that for $a, b, c \in A$ with $b, c \leq a$ we have $b \vee c=p(a, b, c)$. Define $\varphi: \operatorname{Con}(\mathbf{L}) \rightarrow \operatorname{Con}(\mathbf{A})$ by restriction to A. Then $\varphi$ is surjective by filtrality. To verify that $\varphi$ is an order isomorphism and hence a lattice isomorphism, it is enough to show that for $\theta_{1}, \theta_{2} \in \operatorname{Con}(\mathbf{L})$ we have

$$
\theta_{1} \subseteq \theta_{2} \quad \text { if and only if } \quad \varphi\left(\theta_{1}\right) \subseteq \varphi\left(\theta_{2}\right) .
$$

The left-to-right implication is trivial. For the other, assume $\varphi\left(\theta_{1}\right) \subseteq \varphi\left(\theta_{2}\right)$ and suppose that $\left(\ell, \ell^{\prime}\right) \in \theta_{1}$. Without loss of generality, $\ell \leq \ell^{\prime}$. Write $\ell=a_{1} \vee \ldots \vee a_{k}$ and $\ell^{\prime}=\ell \vee a_{k+1} \vee \ldots \vee a_{n}$, where $a_{i} \in A$. Proceeding by induction, we may consider just the case $n=k+1$. The lattice interval $\left[\ell, \ell^{\prime}\right]$ is a transpose of $\left[\ell \wedge a_{k+1}, a_{k+1}\right]$, so we have $a_{k+1} \theta_{1} \ell \wedge a_{k+1}=\left(a_{k+1} \wedge a_{1}\right) \vee \ldots \vee\left(a_{k+1} \wedge a_{k}\right)$, which is in $A$ by the bounded join property with bound $a_{k+1}$. But since $\varphi\left(\theta_{1}\right) \subseteq \varphi\left(\theta_{2}\right), a_{k+1} \theta_{2} \ell \wedge a_{k+1}$ as well, and by transposition $\left(\ell, \ell^{\prime}\right) \in \theta_{2}$. Thus $\theta_{1} \subseteq \theta_{2}$ and $\varphi$ is a lattice isomorphism, as claimed.

It follows that congruence lattices of algebras in $\mathcal{P}$ are isomorphic to congruence lattices of algebras in $\mathcal{D}$. The reverse is also true, since if $\mathbf{L}$ is a distributive lattice and $\mathbf{A}$ is the reduct of $\mathbf{L}$ itself to $\mathcal{P}$, then $\varphi$ is the identity map and $\operatorname{Con}(\mathbf{L})=\operatorname{Con}(\mathbf{A})$.

\section{Acknowledgement}

We thank Keith Kearnes for his valuable suggestions regarding the proof of Theorem 2.4. 


\section{References}

[1] M. E. Adams and H. A. Priestley, 'Equational bases for varieties of Ockham algebras', Algebra Universalis 32 (1994), 368-397.

[2] P. Agliano and K. A. Baker, 'Idempotent discriminators', Technical report (Univ. of Siena, 1997).

[3] — 'Two-generated varieties', Technical report (Univ. of Siena, 1998).

[4] K. A. Baker, 'Primitive satisfaction and equational problems for lattices and other algebras', Trans. Amer. Math. Soc. 190 (1974), 125-150.

[5] _ . 'Finite equational bases for finite algebras in a congruence-distributive equational class', Adv. Math. 24 (1977), 207-243.

[6] J. Berman, 'Distributive lattices with an additional unary operation', Aequationes Math. 16 (1977), $165-171$

[7] W. J. Blok, P. Köhler and D. Pigozzi, 'On the structure of varieties with equationally definable principal congruences II', Algebra Universalis 18 (1984), 334-379.

[8] W. J. Blok and D. Pigozzi, 'On the structure of varieties with equationally definable principal congruences I', Algebra Universalis 15 (1982), 195-227.

[9] ___, 'A finite basis theorem for quasivarities', Algebra Universalis 13 (1986), 1-13.

[10] — 'On the structure of varieties with equationally definable principal congruences III', Algebra Universalis 32 (1994), 545-608.

[11] —_, 'On the structure of varieties with equationally definable principal congruences IV', Algebra Universalis 31 (1994), 1-35.

[12] S. Bulman-Fleming and H. Werner, 'Equational compactness in quasi-primal varieties', Algebra Universalis 7 (1977), 33-46.

[13] S. Burris and H. P. Sankappanavar, A course in universal algebra (Springer, New York, 1981).

[14] W. H. Cornish, Antimorphic action. Categories of algebraic structures with involutions or antiendomorphisms, R\&E Research and Exposition in Mathematics, 12 (Helderman, Berlin, 1986).

[15] G. Gratzer, Universal algebra, second edition (Springer, New York, 1979).

[16] R. Magari, 'Varietà a quozienti filtrali', Ann. Univ. Ferrara, Sez. VII 14 (1969), 5-20.

[17] — 'The classification of idealizable varieties', J. Algebra 26 (1973), 152-165.

[18] R. McKenzie, G.McNulty and W. Taylor, Algebras, lattices, varieties, vol. I (Wadsworth and Brooks/Cole, Belmont, 1987).

Dipartimento di Matematica

Via del Capitano 15

53100 Siena

Italy

e-mail: agliano@unisi.it
Department of Mathematics

UCLA

Los Angeles CA 90095-1555

USA

e-mail: baker@math.ucla.edu 